\section{A computer program for Likert format questionnaire analysis}

\author{
RICHARD F. ANTONAK \\ Department of Education, University of New Hampshire \\ Durham, New Hampshire 03824
}

On college and university campuses, Likert format questionnaires are used for student evaluation of diverse aspects of courses, often with multiple items grouped into sets with a common referent (e.g., instructor, assignments, presentations, etc.). Contrary to summated rating scales which yield a total score to place a respondent somewhere on a continuum of agreement-disagreement toward the attitude being measured (Kerlinger, 1973), course evaluation questionnaires are typically analyzed to obtain respondent group means to each item or to sets of items. Moreover, the instructor examines the number and percentage of respondents selecting each alternative and makes comparisons among items within a set and between sets of items to determine relative strengths and weaknesses of the course.

While subprograms of larger statistical analysis packages, such as SPSS (Nie, Hull, Jenkins, Steinbrenner, \& Bent, 1975), will perform analyses such as those described, they are often inappropriate because they (1) require a computer system large enough to support the entire package, (2) cannot perform an analysis of individual items and sets of items simultaneously, and (3) generate more output than is necessary while requiring extensive variable and parameter definition.

The program described in this paper performs the analyses typically sought for Likert format questionnaires (such as course evaluations) while overcoming the limitations imposed by larger generalized statistical analysis packages.

Input. The job deck consists of the following: title cards, a problem parameters card, item group specification card, item group label card(s), optional question and response choice label cards, a data format card, data deck, and a last card. The first three cards describe the problem in a text which is reproduced on the output. The problem parameters card indicates the number of respondents, the population size, the number of item sets, and user preference for optional alphanumeric labeling. The next card specifies the number of items in each set (or the total number of items if they are ungrouped) and is followed by item group labels (one per card to a maximum of 80 characters). If the user selects alphanumeric output labeling, the next cards contain the questions (one per card to a maximum of 80 characters) and the response alternatives (one per card to a maximum of 20 characters) in the same order in which they appear on the questionnaire. The data format card is an I-type variable-format card which indicates the location of the respondent ID (maximum of five digits) and question responses on the subsequent data cards. Each of the data cards must contain the data for one respondent; however, the use of more than one card per respondent is permitted. If the user wishes to terminate the program, the last card must contain the word END punched in columns $1-3$. On the other hand, if the user wishes to analyze another set of data, the last card is blank and the job deck is arranged sequentially (as described above), beginning with the title cards.

Output. The printed output includes: (1) the titling information, (2) a respondent by question response matrix, (3) item response distribution statistics organized by item sets, (4) distribution statistics for the item sets, and (5) a hierarchical reordering of all item and item set statistics. The item distribution statistics include the number and percentage of individuals responding to the item (and to the questionnaire) who selected each alternative, the number and percentage of respondents who omitted the item, a histogram, and the item mean, variance, and standard deviation. The item set analysis includes the set mean, variance, and standard deviation adjusted for missing data points and a presentation in ascending order by the means of the statistics for each item within the set. Finally, all item and item set statistics are presented in a similar ascending order.

Computer and Language. The program is written in FORTRAN-10 for processing by computers in the DEC-10 series and is adaptable to CDC and IBM systems. Well documented, the program has variable names that are mnemonic to facilitate modification by the user.

Restrictions. The program has the following restrictions: (1) there cannot be more than 500 respondents, (2) there cannot be more than 100 items, (3) the maximum number of item sets is 26 , and (4) the number of item response alternatives, while variable, cannot exceed seven.

Avallability. Copies of this paper and a source listing, which includes data and printed output for a sample analysis, may be obtained without charge by writing to Richard F. Antonak, Department of Education, University of New Hampshire, Durham, New Hampshire 03824.

\section{REFERENCES}

KERLINGER, F. N. Foundations of behavioral research (2nd ed.). New York: Holt, Rinehart, \& Winston, 1973.

Nie, N. H., Hull, C. H., Jenkins, J. G., Steinbrenner, K., \& BENT, D. H. Statistical package for the social sciences (2nd ed.). New York: McGraw-Hill, 1975. 